\title{
Chemical Abundances of Cold Stars in Binary Nuclei of Abell 35-type Planetary Nebulae
}

\author{
G. Jasniewicz ${ }^{1,2}$, F. Thévenin ${ }^{3}$ and A. Acker ${ }^{1}$ \\ ${ }^{1}$ Observatoire de Strasbourg; ${ }^{2}$ GRAAL Montpellier; ${ }^{3}$ Observatoire de Nice
}

Jasniewicz et al. (1994) have announced and started in 1994 a program of chemical analysis of the G-stars at the centre of Abell 35-type planetary nebulae. First results concerning BD $-22^{\circ} 3467$ and HD 112313 located respectively at the centre of Abell 35 and LoTr 5 have been published by Jasniewicz et al. (1996) and Thévenin \& Jasniewicz (1996).

We emphasize here the most appreciable result: spectra of both stars display an iron abundance nearly solar $([\mathrm{Fe} / \mathrm{H}] \approx-0.20 \pm 0.30 \mathrm{dex})$, an abundance of the carbon $([\mathrm{C} / \mathrm{Fe}] \approx$ $+0.00 \pm 0.40 \mathrm{dex}$ ) normal with regard to the Fe abundance, and a clear enhancement in barium $([\mathrm{Ba} / \mathrm{H}] \approx+0.50 \pm 0.30 \mathrm{dex})$. The BaII line at $4554 \AA$ is especially strengthened in the spectrum of HD 112313. For HD 112313, we have also found a significant overabundance of other s-processed elements such as strontium $([\mathrm{Sr} / \mathrm{Fe}] \approx+0.80 \pm 0.30 \mathrm{dex})$ and yttrium $([\mathrm{YFe}] \approx+0.40 \pm 0.30 \mathrm{dex})$.

We suggest that the observed enhancement of the barium element in BD $-22^{\circ} 3467$ and HD 112313 is caused by the transfer of s-process-overabundant material from the former AGB star which is now the hot star (discovered by means of IUE spectra) of the binary system. Several evolutionary models can explain the formation of the Abell 35 type planetary nebula nuclei via binary interactions: wind accretion, wind exposure, stable Roche lobe overflow and common-envelope ejection. The high equatorial rotational velocity of BD-22 $3467\left(v \sin i=55 \pm 5 \mathrm{~km} \mathrm{~s}^{-1}\right)$ and HD $112313\left(v \sin i=60 \pm 5 \mathrm{~km} \mathrm{~s}^{-1}\right)$ could be explained by two different scenarios: spin-up during a common envelope interaction (Bond 1993) or during a wind-accretion phase (Jeffries \& Stevens 1996). In the first case, the actual binary should be a close binary whilst in the second case the binary could be a wide one. Our whole set of data concerning Abell 35 and LoTr 5 obtained during 10 years is consistent with the second scenario (see Thévenin \& Jasniewicz 1996).

\section{REFERENCES}

Bond H.E., 1993, In: Barstow M.A. (ed) White Dwarfs: Advances in Observation and Theory. Kluwer, Dordrecht, p. 85

Jasniewicz G., Thévenin F., Acker A., 1994, Chemical abundances in binary nuclei of evolved planetary nebulae. In: Acker A. \& Köppen J. (eds), Planetary Nebulae, Workshop "Col de Steige". Observatoire de Strasbourg, GdR 968, p.46

Jasniewicz G., Thévenin F., Monier R., Skiff B., 1996, Astron. Astrophys., 307, 200

Jeffries R.D., Stevens I.R., 1996, Mon. Not. R. Astron. Soc., 279, 180

Thévenin F., Jasniewicz G., 1996, Astron. Astrophys., in press 